\title{
EDITORIAL
}

\section{Valuing new technologies}

Society benefits from new health technologies which improve health and welfare outcomes; increase the productivity of the health sector and reduce the costs of health production. Nevertheless, innovative new technologies are by necessity associated with several unknowns in their early phases. The uncertainty is faced by developers of new health technologies in research institutions and in the health industry as well as health care systems who are the future payers. Some innovative new technologies will change treatment paradigms and move the health production possibility curve to a new level, while others will contribute to the continuous improvement of available technologies. The true value and degree of innovation of a new technology may be difficult to assess in its early phase before long-term effectiveness data on larger patient groups become available. There may also be a concern for potential unforeseen side-effects. Since the 1960s, several health sector institutions have been established with an aim of examining the efficacy and safety of new technologies, especially for pharmaceuticals. Still, the uncertainty inherent in new technologies gives incentives for slow introduction and reduces the willingness-to-pay for buyers, all else equal. Developers, facing these market realities, will invest less in research compared to a situation with less uncertainty.

Strategies and methods for valuing new health technologies have become especially important for medicines where regulatory agencies have strict procedures for assessment of efficacy and safety of new drugs. Approval of a new drug is a necessary step but further information on its cost-effectiveness is essential for payors. The assessment of value of a new medicine in this early phase will for natural reasons rely on limited data from clinical trials. Economists have developed strategies and guidelines for good practice for economic evaluations. The question in an economic evaluation will then be "Is this new therapy/drug cost-effective at the proposed price compared a relevant treatment alternative, typically the current standard of care treatment for the patient group?". In reality, this question contains caveats such as "based on current evidence using data generated in experimental settings where the sample size is determined for the establishment efficacy in key clinical variables and a selection of patients without comorbidities to facilitate conclusions" and "provided that it is reasonable to use epidemiological data on long-term natural development of the disease". In principle, postponing the approval of reimbursement until more information on efficacy, effectiveness and safety becomes available is possible, but comes at the cost of not realised health gains and delayed benefits to patients as a group.

This special issue of the Nordic Journal of Health Economics presents six papers on the theme "The value of new technologies". The established institutions and regulatory framework for the evaluation of new medicines in terms of safety, efficacy and costeffectiveness renders medicines a leading position in terms of published analyses for valuation of new technologies for health. The first paper of this special issue presents an overview of a selection of studies that have estimated the long-term and life-cycle value of new medicines that have been introduced in Sweden. It is written by the authors of the final report of the research programme at the Centre for Business and Policy Studies on the theme The Value of New Medicines, Katarina Steen Carlsson and Bengt Jönsson.

Two papers by Frank R Lichtenberg analyse of the impact of drug innovation on longevity. Using data on use of cardiovascular drugs in Switzerland, Lichtenberg shows that people who used newer cardiovascular drugs had longer time till death than people who 
used older cardiovascular drugs when the analysis controlled for both type of prescription and individual characteristics. The second paper is an overview and discussion of methods for analysing observational data and studies that have sought to measure the impact of biomedical innovation on the longevity and health of Americans and other populations during recent decades.

In a debate paper, Thomas $\mathbf{J}$ Philipson raises a concern for methodological issues regarding the use of cost-effectiveness as a decision criterion for payers of health care. Philipson illustrates his arguments with examples where multiple, on their own laudable, goals could be conflicting and maximization of one may only be achieved at the expense of other goals. He concludes that concerns for the issues regarding static- and dynamic efficiency imply that much more research needs to be done on the implications of using CEbased reimbursement policies for technology adoption.

Joakim Ramsberg and Martin Neovius present additional ways of establishing efficacy, safety and cost-effectiveness besides the often costly randomized clinical trials. They give a background and overview of recent methodological advances conducting clinical and cost-effectiveness research using registers and electronic health records. The authors find that the pragmatic randomized trial, enriched with routinely collected register data, may be an effective solution for creating fast and much less costly evidence. However, these studies must also include variables relevant for health economic evaluations.

A paper by Kasper Munk Johannesen and Martin Henriksson discuss the incentives in the market for innovative technologies for health. They draw attention to a key challenge which may be especially visible for innovative medicines: research and development investments are uncertain and may take long time to materialize into patient values.

For the editors of the Special Issue of Value of New Technologies

Katarina Steen Carlsson 